\title{
Mutations of RAS/RAF Proto-oncogenes Impair Survival After Cytoreductive Surgery and HIPEC for Peritoneal Metastasis of Colorectal Origin
}

\author{
Marcel André Schneider, MD, ${ }^{*}$ Janina Eden, MD, † Basile Pache, MD, $\ddagger$ Felix Laminger, MD, $\S$ \\ Victor Lopez-Lopez, MD, PhD, || Thomas Steffen, MD, $\dagger$ Martin Hübner, MD, $\ddagger$ Friedrich Kober, MD, $\S$ \\ Sebastian Roka, MD, $\S$ Pedro Cascales Campos, MD, PhD, || Lilian Roth, MD, ${ }^{*}$ Anurag Gupta, PhD, ${ }^{*}$ \\ Alexander Siebenhüner, MD, Vahan Kepenekian, MD,\# Guillaume Passot, MD, PhD,\# \\ Philippe Gertsch, MD, ${ }^{*}$ Olivier Glehen, MD, PhD,\# and Kuno Lehmann, MD, PhD*
}

Background: Adequate selection of patients with peritoneal metastasis (PM) for cytoreductive surgery (CRS) and hyperthermic intraperitoneal chemotherapy (HIPEC) remains critical for successful long-term outcomes. Factors reflecting tumor biology are currently poorly represented in the selection process. The prognostic relevance of $R A S / R A F$ mutations in patients with PM remains unclear.

Methods: Survival data of patients with colorectal PM operated in 6 European tertiary centers were retrospectively collected and predictive factors for survival identified by Cox regression analyses. A simple point-based risk score was developed to allow patient selection and outcome prediction.

Results: Data of 524 patients with a median age of 59 years and a median peritoneal cancer index of 7 (interquartile range: 3-12) were collected. A complete resection was possible in 505 patients; overall morbidity and 90-day mortality were $50.9 \%$ and $2.1 \%$, respectively. PCI [hazard ratio (HR): 1.08], N1 stage (HR: 2.15), N2 stage (HR: 2.57), G3 stage (HR: 1.80) as well as $K R A S$ (HR: 1.46) and $B R A F$ (HR: 3.97) mutations were found to significantly impair survival after CRS/HIPEC on multivariate analyses. Mutations of RAS/ $R A F$ impaired survival independently of targeted treatment against EGFR. Consequently, a simple point-based risk score termed BIOSCOPE (BIOlogical Score of COlorectal PEritoneal metastasis) based on PCI, N-, G-, and RAS/ $R A F$ status was developed, which showed good discrimination [development area under the curve $(\mathrm{AUC})=0.72$, validation $\mathrm{AUC}=0.70]$, calibration $(P=$ $0.401)$ and allowed categorization of patients into 4 groups with strongly divergent survival outcomes.

From the *Department of Surgery and Transplantation, University Hospital of Zurich, Zurich, Switzerland; †Department of Surgery, Cantonal Hospital of St. Gallen, St. Gallen, Switzerland; †Department of Surgery, Lausanne University Hospital (CHUV), Lausanne, Switzerland; §Department of Surgery, Center for Peritoneal Carcinomatosis, Hanusch-Krankenhaus, Vienna, Austria; |Department of Surgery, Hospital Clínico Universitario Virgen de la Arrixaca, Murcia, Spain; Department of Medical Oncology, University Hospital of Zurich, Zurich, Switzerland; and \#Department of Digestive Surgery and Surgical Oncology, Université Hospital Lyon, Lyon, France.

The authors report no conflicts of interest.

Supplemental digital content is available for this article. Direct URL citations appear in the printed text and are provided in the HTML and PDF versions of this article on the journal's Web site (www.annalsofsurgery.com).

Thomas Steffen, Martin Hübner, Philippe Gertsch and Kuno Lehmann: For the Swiss Peritoneal Cancer Group of the Swiss Society for Visceral Surgery, Switzerland.

Reprints: Kuno Lehmann, MD, PhD, Department of Surgery and Transplantation, UniversityHospital Zurich, Raemistrasse 100, CH-8091 Zurich, Switzerland. E-mail: Kuno.Lehmann@usz.ch.

Copyright (C) 2018 Wolters Kluwer Health, Inc. All rights reserved.

ISSN: 0003-4932/18/26805-0845

DOI: $10.1097 /$ SLA.0000000000002899
Conclusion: RAS/RAF mutations impair survival after CRS/HIPEC. The novel BIOSCOPE score reflects tumor biology, adequately stratifies longterm outcomes, and improves patient assessment and selection.

Keywords: colorectal carcinoma, cytoreductive surgery (CRS), hyperthermic intraperitoneal chemotherapy (HIPEC), KRAS/NRAS/BRAF, peritoneal metastasis

(Ann Surg 2018;268:845-853)

$P$ eritoneal metastasis (PM) from colorectal carcinoma (CRC) carries a worse prognosis than other isolated distant metastases of CRC. ${ }^{1}$ Over the last years, the combination of effective systemic therapy in combination with cytoreductive surgery (CRS) and hyperthermic intraperitoneal chemotherapy (HIPEC) improved cancerspecific survival (CSS) in patients with PM. ${ }^{2}$ Although it has been shown that this invasive procedure can be performed with acceptable mortality and morbidity rates, ${ }^{3}$ selection of patients is critical to avoid unnecessary procedures in patients, who do not benefit from CRS/HIPEC. ${ }^{4}$ Multiple factors influence successful postoperative long-term oncological outcomes, for example, the completeness of resection $^{5}$ or the absence of major postoperative complications. ${ }^{6,7}$

However, the role of tumor biology and $R A S / R A F$ mutations in the context of PM remains unclear. RAS/RAF proteins work as downstream secondary messengers of the epidermal growth factor receptor (EGFR) which is expressed on $85 \%$ of patients with metastatic CRC. ${ }^{8} R A S$ mutations belong to the hallmark characteristics of CRC and affect between $30 \%$ to $50 \%$ (KRAS) and 3\% to 5\% (NRAS) of patients with metastatic CRC, ${ }^{8,9}$ whereas mutations of $B R A F$ are found in $3 \%$ to $10 \%{ }^{9-13}$ and are mutually exclusive with RAS mutations. ${ }^{13,14}$ Antibodies blocking EGFR (Cetuximab and Panitumumab) and small-molecule inhibitors (Erlotinib and Gefitinib), preventing intracellular tyrosine kinase activation, were developed to counteract activation of RAS/RAF proteins and their downstream targets. ${ }^{15}$ They modestly improve overall- and recurrence-free survival (RFS), when given alone or in combination with standard regimens. ${ }^{16,17}$ In patients with PM of colorectal origin, the role of $R A S / R A F$ mutations has not been examined and might be helpful to predict survival after CRS/HIPEC in combination with other prognostic factors. Available scores ${ }^{18}$ and nomograms ${ }^{19}$ are complex, and show a moderate ability to predict the outcome of patients after CRS/HIPEC. ${ }^{20}$ Currently, there is no preoperative scoring system which incorporates tumor biology as a prognostic factor and accurately predicts oncological outcomes. The primary aim of our current analyses was therefore to investigate the role $R A S / R A F$ on CSS and RFS in patients undergoing CRS/HIPEC. 
Secondary goal was to develop a sore based on tumor biology-related factors to adequately predict oncological outcomes.

\section{METHODS}

\section{Patients and Ethics}

The study was approved by the lead ethics committee of the cantonal authorities in Zurich, Switzerland (KEK-ZH-Nr. 201701656) and the respective local authorities (Supp. Info. 1, http:// links.lww.com/SLA/B449). Data of patients with PM from colorectal cancer from 6 European centers (Zurich, St. Gallen, and Lausanne, Switzerland; Lyon, France; Murcia, Spain; and Vienna, Austria) were collected and analyzed. Latest follow-up data including dates of death or recurrences and further oncological therapies were obtained from the hospital databases or by directly contacting patients or oncologists.

\section{Treatment}

Patients were presented at multidisciplinary tumor boards at the respective institutions and selected for CRS/HIPEC after clinical workup and exclusion of extra abdominal tumor manifestations by ${ }^{18}$ FDG-PET/CT or thoracic-abdominal CT. Patients received standard of care pre- and postoperative chemotherapy according to international guidelines, based on leucovorin, 5-fluorouracil, irinotecan, and/or oxaliplatin, in combination with targeted therapies (monoclonal antibodies or small molecule inhibitors targeting VEGF $\alpha /$ angiogenesis or EGFR) where appropriate. CRS/HIPEC was performed by experienced and trained surgeons in all centers as specified elsewhere. ${ }^{21,22}$ Mitomycin C sole [15-30 mg/m $\mathrm{m}^{2}$ Body Surface Area (BSA)] or in combination with doxorubicin $\left(15 \mathrm{mg} / \mathrm{m}^{2} \mathrm{BSA}\right)$ at $42^{\circ} \mathrm{C}$ for 90 minutes or oxaliplatin (300-400 mg/m $\mathrm{m}^{2} \mathrm{BSA}$ ) as single agent at $43^{\circ} \mathrm{C}$ for 30 minutes were used for HIPEC. A procedure was defined as curative, if radical CRS (CC-Score $0,{ }^{23}$ no macroscopic residual tumor) followed by HIPEC could be performed.

\section{Pathology}

PCI was calculated after careful inspection following laparotomy at the beginning of CRS/HIPEC. Information on TNM-stage, Gstage, histology, and $R A S / R A F$ status was retrieved from pathology reports of the operating hospital or referral letters and previous pathology reports.

\section{Statistical Analyses and Risk Score Development}

All statistical analyses were performed with R (Supp. Info. 2, http://links.lww.com/SLA/B449). Continuous data are reported as median $(\mathrm{m})$ and interquartile range (IQR). Wilcoxon rank-sum test or Fisher exact test were used to compare medians, resp. frequencies between groups. Statistical significance was defined as $P<0.05$. CSS and RFS were calculated with Kaplan-Meier estimates from the date of the operation until date of cancer-related death, disease progression or date of last follow up in months. Mantel-Cox logrank test was used to test for differences between survival times with Bonferroni correction for multiple comparisons. A total of 1000 bootstrapped samples were used to calculate $95 \%$ confidence intervals (CIs) of all measures or standard error (SE) of regression coefficients. Uni- and multivariate analyses were performed with Cox's proportional hazard regression models; predictors with a $P$ value of less than 0.1 were included in the multivariate model. Missing values of categorical data were grouped into a category "Non-determined" for Cox regression and risk score development with $\beta$-coefficients of respective groups not reported; no exclusion due to missing data nor data imputation was performed. ${ }^{24}$

The risk score was developed according to the TRIPOD guidelines $^{25}$ by splitting the patient cohort randomly into a development $(70 \%-75 \%$ of patients) and validation $(25 \%-30 \%$ of patients) cohort (type 2A). A Cox regression model of factors identified in multivariate analyses was afterward fitted to the development cohort. The discriminative ability of the model was assessed in the development and validation cohort with receiver-operating characteristic (ROC) curves and corresponding area under the curve (AUC) values of 1000 bootstrapped samples at fixed time points, whereas Nagelkerke $R^{2}$ values served as parameter of the explained variability of the model. Calibration of observed survival frequencies and predicted survival probability of the model were performed with the jackknife pseudo-value method and evaluated by the Grønnesby and Borgan goodness-of-fit test. ${ }^{26} \mathrm{~A}$ shrinkage factor was calculated via 10-fold internal cross validation on the development cohort to reduce the risk of model over-fitting. Consequently, whole integers were assigned to risk factor levels by dividing shrinkage-adjusted regression $\beta$-coefficients by a predetermined constant $\mathrm{B}$ according to previous studies. ${ }^{27,28}$ The derived score was afterward applied to both cohorts for internal validation.

\section{RESULTS}

\section{Patient Characteristics}

Data of 524 patients operated between January 2005 and December 2017 were collected and analyzed. Table 1 summarizes details of patient demographics, clinicopathological, and treatment characteristics.

\section{Surgical and Oncological Treatment}

Most patients $(78.63 \%)$ received preoperative irinotecan- or oxaliplatin-based systemic chemotherapy regimens; $40 \%$ of patients received additional anti-EGFR or anti-VEGF treatment. A complete resection was reported in $96.37 \%$ of patients with a median PCI of 7 (IQR 3-12). The overall recorded complication rate after CRS/ HIPEC was $51 \%$, whereas major complications, defined according to the Clavien-Dindo classification as grade $\geq$ IIIA, ${ }^{29}$ occurred in $16.9 \%$ of patients. The reoperation rate was $10.2 \%$ and 11 patients died during the postoperative course. Postoperative systemic chemotherapy was performed in $61 \%$ of patients during follow-up.

\section{Patient Survival and Prognostic Factors}

The latest follow-up data were received on the January 31, 2018. Mean follow-up at the time of analysis was 27 months, with 204 (38.39\%) patients succumbing to their disease and 375 (71.56\%) patients experiencing disease progression. Patients after complete CRS/HIPEC showed an mCSS of 45 months (CI: 38-52) and mRFS of 12 months (CI: 11-13, Supp. Fig. 1, http://links.lww.com/SLA/ B449). All further analyses were performed on patients after complete CRS/HIPEC without lethal postoperative complication $(n=494)$.

In multivariate analysis, PCI (HR 1.08 per additional point), N1-stage (HR 2.15), N2-stage (HR 2.57), and G3-stage (HR 1.80) were confirmed as factors predicting impaired CSS after radical CRS/HIPEC (Table 2). KRAS (HR 1.46) and BRAF (HR 3.97) mutations were identified as novel factors for impaired survival (Fig. 1A). PCI (HR 1.05), N2-stage (HR 1.55), and KRAS mutations (HR 1.42) were risk factors for shorted RFS (Fig. 1B, Supp. Table 1, http://links.lww.com/SLA/B449).

\section{RAS/RAF Mutational Status in the Setting of Multimodal Therapy}

To determine if the observed differences in CSS between RAS/ $R A F$ wild-type and mutated patients are due to intrinsic tumor biology or related to therapy with targeted therapies against EGFR, patients with known $R A S / R A F$ status $(\mathrm{n}=378)$ were divided in 3 groups: patients with $R A S / R A F$ mutations $(\mathrm{n}=186)$, patients with wild-type $R A S / R A F$ which received chemotherapy without anti-EGFR treatment 
TABLE 1. Baseline Patient Demographic, Clinicopathological, and Treatment Characteristics of 524 Patients Undergoing CRS/HIPEC for PM of Colorectal Origin

\begin{tabular}{|c|c|}
\hline Variable & n $(\%)$ \\
\hline \multicolumn{2}{|l|}{$\overline{\operatorname{Sex}}$} \\
\hline Male & $257(49.1 \%)$ \\
\hline Female & $267(50.9 \%)$ \\
\hline Age, yr & $59(50-66)$ \\
\hline \multicolumn{2}{|l|}{ T-stage of the primary tumor } \\
\hline T3 & $119(22.7 \%)$ \\
\hline $\mathrm{T} 4$ & $213(40.7 \%)$ \\
\hline Tx/not available & $192(36.6 \%)$ \\
\hline \multicolumn{2}{|l|}{$\mathrm{N}$-stage of the primary tumor } \\
\hline No & $85(16.2 \%)$ \\
\hline N1 & $119(22.7 \%)$ \\
\hline $\mathrm{N} 2$ & $131(25.0 \%)$ \\
\hline Nx/not available & $189(36.1 \%)$ \\
\hline \multicolumn{2}{|l|}{ G-stage/histological grading } \\
\hline G1 & $90(17.2 \%)$ \\
\hline $\mathrm{G} 2$ & $211(40.3 \%)$ \\
\hline G3 & $88(16.8 \%)$ \\
\hline $\mathrm{Gx} /$ not available & $135(25.7 \%)$ \\
\hline \multicolumn{2}{|l|}{ Histological subtype } \\
\hline Intestinal adenocarcinoma & $401(76.5 \%)$ \\
\hline Mucinous adenocacinoma & $62(11.8 \%)$ \\
\hline Signet-ring cell adenocarcinoma & $37(7.1 \%)$ \\
\hline Not available & $24(4.6 \%)$ \\
\hline \multicolumn{2}{|l|}{ Tumor localization } \\
\hline Caecum & $57(10.9 \%)$ \\
\hline Colon ascendens & $123(23.5 \%)$ \\
\hline Colon transversum & $22(4.2 \%)$ \\
\hline Colon descendens & $82(15.6 \%)$ \\
\hline Sigmoid colon & $195(37.2 \%)$ \\
\hline Rectum & $36(6.9 \%)$ \\
\hline Not available & $9(1.7 \%)$ \\
\hline \multicolumn{2}{|l|}{ Appearance of PM } \\
\hline Synchronous & $283(54.0 \%)$ \\
\hline Metachronous & $241(46.0 \%)$ \\
\hline \multicolumn{2}{|l|}{ RAS/RAF mutations } \\
\hline Wild-type & $202(38.5 \%)$ \\
\hline KRAS mutation & $154(29.5 \%)$ \\
\hline$N R A S$ mutation & $24(4.6 \%)$ \\
\hline$B R A F$ mutation & $20(3.8 \%)$ \\
\hline Not available & $154(29.4 \%)$ \\
\hline \multicolumn{2}{|l|}{ Center } \\
\hline Lausanne & $15(2.7 \%)$ \\
\hline Lyon & $363(69.3 \%)$ \\
\hline Murica & $25(5.0 \%)$ \\
\hline St. Gallen & $33(6.3 \%)$ \\
\hline Vienna & $17(3.3 \%)$ \\
\hline Zurich & $70(13.4 \%)$ \\
\hline \multicolumn{2}{|l|}{ PCI } \\
\hline PCI $0-5$ & $212(40.5 \%)$ \\
\hline PCI 6-10 & $163(31.1 \%)$ \\
\hline PCI $11-15$ & $55(10.5 \%)$ \\
\hline PCI $16-20$ & $56(10.6 \%)$ \\
\hline PCI $21-25$ & $29(5.4 \%)$ \\
\hline PCI $26-30$ & $7(1.3 \%)$ \\
\hline PCI $31-35$ & $1(0.2 \%)$ \\
\hline PCI $36-39$ & $1(0.2 \%)$ \\
\hline Median PCI (+IQR) & $7(3-12)$ \\
\hline \multicolumn{2}{|l|}{ HIPEC regimen } \\
\hline Oxaliplatin & $266(50.8 \%)$ \\
\hline Mitomycin C/Doxorubicin & $44(8.4 \%)$ \\
\hline Mitomycin & $171(32.6 \%)$ \\
\hline Other/not documented & $43(8.2 \%)$ \\
\hline
\end{tabular}

(Continued)

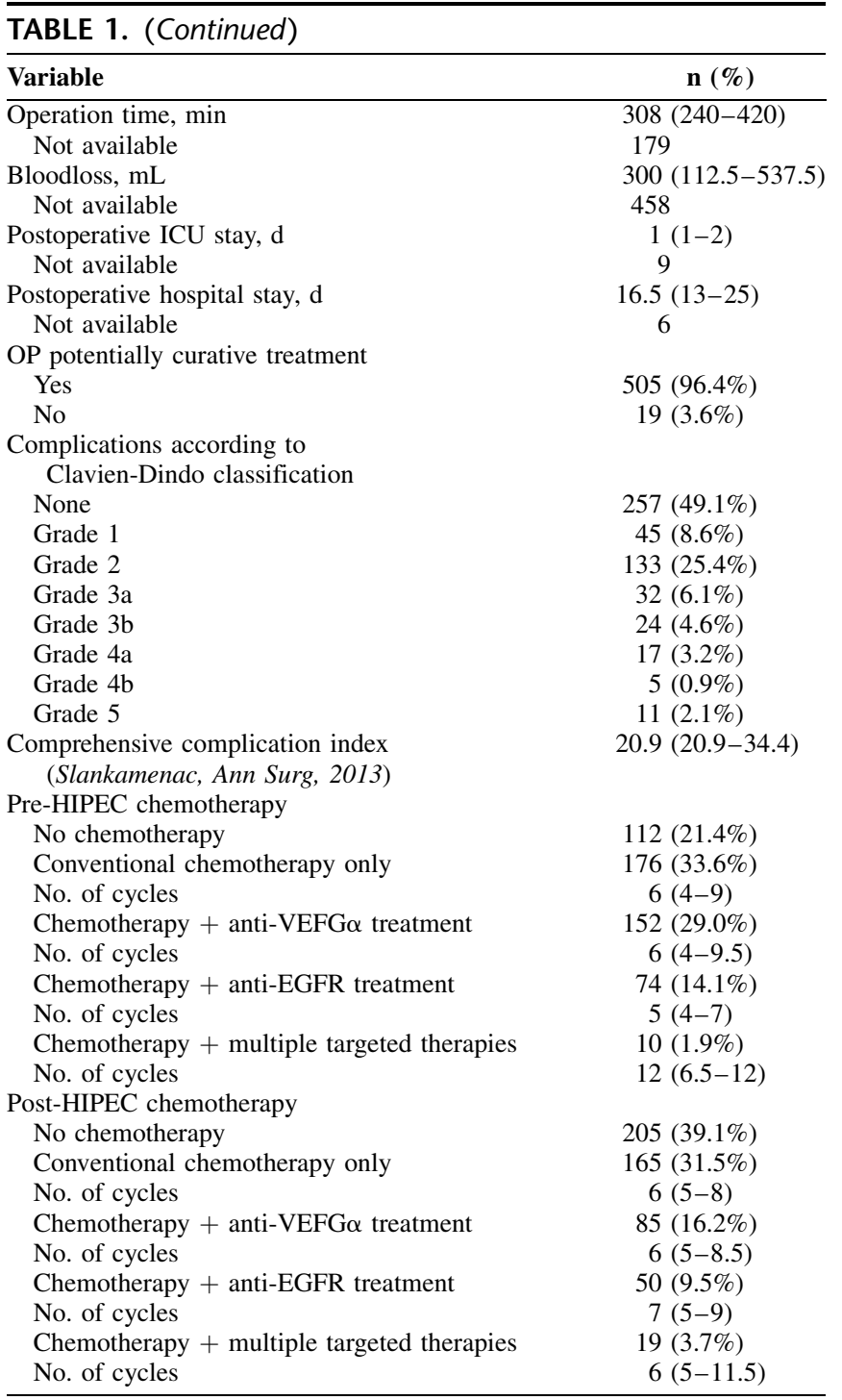

CRS indicates cytoreductive surgery; HIPEC, hyperthermic intraperitoneal chemotherapy; PM, peritoneal metastasis.

Continuous variables are shown as median and interquartile range.

$(\mathrm{n}=115)$, and patients with wild-type $R A S / R A F$ treated with antiEGFR treatment $(\mathrm{n}=77)$ (Fig. 1C). Interestingly, patients with wildtype $R A S / R A F$ had improved survival regardless of treatment with $(P=$ $0.047)$ or without $(P=0.028)$ targeted anti-EGFR treatment compared with patients with RAS/RAF mutations, indicating a mutation-caused rather than treatment-related effect on survival.

\section{Development of a New Risk Score for Patient Selection Based on Tumor Biology}

Randomized splitting allocated 358 (72.4\%) patients into the development cohort and $136(27.6 \%)$ patients into the validation cohort (Fig. 2A). A Cox regression model with the four identified predictive factors PCI, N-, G-, and $R A S / R A F$ mutation status was consequently fitted to the development cohort, which discriminated survival at 36 months with a AUC-value of 0.72 in the development cohort (Fig. 2B) and 0.70 in the validation cohort (Fig. 2C). The good discrimination of $>0.7$ was consistent over all time points tested 
TABLE 2. Uni- and Multivariate Analysis of Factors Predicting Cancer-specific Survival (CSS) in Patients With PM of Colorectal Origin After Complete CRS/HIPEC $(n=494)$

\begin{tabular}{|c|c|c|c|c|c|c|}
\hline \multirow[b]{2}{*}{ Variable } & \multirow[b]{2}{*}{ n $(\%)$} & \multirow[b]{2}{*}{ Median CSS, mo (95\% CI) } & \multicolumn{2}{|c|}{ Univariate Analysis } & \multicolumn{2}{|c|}{ Multivariate Analysis } \\
\hline & & & Hazard Ratio (95\% CI) & $P$ & Hazard Ratio (95\% CI) & $P$ \\
\hline \multicolumn{7}{|l|}{ Sex } \\
\hline Male & $236(47.8 \%)$ & $50(35-65)$ & & & & \\
\hline Female & $258(52.2 \%)$ & $43(35-53)$ & $1.17(0.87-1.57)$ & 0.284 & & \\
\hline Age (per additional year) & & & $0.98(0.97-0.99)$ & 0.046 & $0.98(0.97-1.00)$ & 0.405 \\
\hline \multicolumn{7}{|l|}{ Year of CRS/HIPEC } \\
\hline $2005-2012$ & $193(39.1 \%)$ & $45(40-50)$ & & & & \\
\hline $2013-2017$ & $301(60.9 \%)$ & $44(34-53)$ & $1.18(0.86-1.63)$ & 0.299 & & \\
\hline \multicolumn{7}{|l|}{ T-stage } \\
\hline $\mathrm{T} 3$ & $113(36.4 \%)$ & $45(26-63)$ & & & & \\
\hline $\mathrm{T} 4$ & $197(63.6 \%)$ & $38(28-48)$ & $1.23(0.85-1.79)$ & 0.259 & & \\
\hline \multicolumn{7}{|l|}{$\mathrm{N}$-stage } \\
\hline No & $80(25.6 \%)$ & $57(51-62)$ & & & & \\
\hline N1 & $111(35.5 \%)$ & $36(26-45)$ & $2.24(1.28-3.95)$ & 0.004 & $2.15(1.18-3.90)$ & 0.011 \\
\hline $\mathrm{N} 2$ & $122(39.0 \%)$ & $36(27-46)$ & $2.91(1.70-4.98)$ & $\leq \mathbf{0 . 0 0 1}$ & $2.57(1.47-4.49)$ & $\leq \mathbf{0 . 0 0 1}$ \\
\hline \multicolumn{7}{|l|}{ G-stage } \\
\hline G1 & $86(23.6 \%)$ & $51(38-65)$ & & & & \\
\hline G2 & $200(55.0 \%)$ & $45(30-60)$ & $0.92(0.62-1.38)$ & 0.714 & $1.09(0.71-1.67)$ & 0.672 \\
\hline G3 & $78(21.4 \%)$ & $31(22-40)$ & $1.84(1.17-2.90)$ & 0.008 & $1.80(1.06-3.05)$ & 0.028 \\
\hline \multicolumn{7}{|l|}{ Histology } \\
\hline Adenocarcinoma & $380(80.5 \%)$ & $47(39-54)$ & & & & \\
\hline Mucinous adenocarcinoma & $61(12.9 \%)$ & $49(23-75)$ & $1.21(0.78-1.89)$ & 0.385 & $1.19(0.69-2.04)$ & 0.526 \\
\hline Singet ring cell & $31(6.6 \%)$ & $34(21-47)$ & $1.86(1.05-3.30)$ & 0.032 & $1.07(0.56-2.04)$ & 0.835 \\
\hline \multicolumn{7}{|l|}{ Localisation } \\
\hline Coecum/ascendens & $171(35.2 \%)$ & $36(29-43)$ & & & & \\
\hline Transversum/descendens & $99(20.4 \%)$ & $77(40-104)$ & $0.59(0.40-0.86)$ & 0.001 & $0.67(0.42-1.04)$ & 0.073 \\
\hline Rectosigmoid & $216(44.4 \%)$ & $51(39-63)$ & $0.73(0.54-0.97)$ & 0.003 & $0.78(0.55-1.09)$ & 0.155 \\
\hline \multicolumn{7}{|l|}{ Temporal appearance of PM } \\
\hline Synchronous & $268(54.3 \%)$ & $41(33-49)$ & & & & \\
\hline Metachronous & $226(45.7 \%)$ & $48(39-57)$ & $0.85(0.63-1.15)$ & 0.302 & & \\
\hline PCI per additional point & & & $1.08(1.06-1.10)$ & $\leq \mathbf{0 . 0 0 1}$ & $1.08(1.06-1.10)$ & $\leq \mathbf{0 . 0 0 1}$ \\
\hline \multicolumn{7}{|l|}{$R A S / R A F$ mutation } \\
\hline Wildtype & $192(50.8 \%)$ & $52(40-65)$ & & & & \\
\hline$K R A S$ mutation & $145(38.4 \%)$ & $38(31-46)$ & $1.45(1.02-2.07)$ & 0.036 & $1.46(1.00-2.12)$ & 0.048 \\
\hline NRAS mutation & $19(5.0 \%)$ & $49(19-80)$ & $1.22(0.65-2.33)$ & 0.533 & $0.88(0.45-1.72)$ & 0.711 \\
\hline$B R A F$ mutation & $22(5.8 \%)$ & $18(9-26)$ & $4.29(2.16-8.51)$ & $\leq \mathbf{0 . 0 0 1}$ & $3.97(1.86-8.44)$ & $\leq \mathbf{0 . 0 0 1}$ \\
\hline \multicolumn{7}{|c|}{ Major complication ( $\geq$ Clavien Dindo classification 3A) } \\
\hline No major complication & $421(85.2 \%)$ & $49(40-57)$ & & & & \\
\hline Major complication & $73(14.8 \%)$ & $38(27-49)$ & $1.53(1.05-2.23)$ & 0.026 & $1.20(0.78-1.84)$ & 0.406 \\
\hline
\end{tabular}

(Supp. Fig. 2A, http://links.lww.com/SLA/B449). Comparison of observed survival frequencies and predicted survival probabilities at 36 months (Fig. 2D) and additional time points (Supp. Fig. 2B and C, http://links.lww.com/SLA/B449) confirmed the good calibration of the model in the development (goodness-of-fit test: $P=0.401$ ) as well as validation cohort (goodness-of-fit test: $P=0.483$ ). Crossvalidation yielded a global shrinkage coefficient of 0.9098 . Using the shrinkage-adjusted $\beta$ regression coefficients for allocation of points to the respective factors levels, the risk score with a range of 0 to 12 points was calculated (Fig. 3A). The resulting score was consequently applied on development and validation cohort and showed a consistent stepwise decrease of mCSS with increasing amount of points (Fig. 3B and C). In a last step, 4 risk groups were defined based on the allocated points. Patients with a risk score of 0 (risk group A) showed excellent long-term outcomes. Patients with minimal (1-3) risk points had a median survival over the average of 43 months of the whole cohort and were defined as risk group B. Patients with intermediate risk scores between 4 and 7 (risk group C) showed an acceptable survival after CRS/HIPEC, whereas patients with risk scores 8 and higher (risk group D) had a dismal long-term outcome (Fig. 3D and E); the score also allowed stratification of PFS in both cohorts to a certain extent (Supp. Fig. 3A and B, http://links. lww.com/SLA/B449).

\section{DISCUSSION}

The present study for the first time identifies mutations of $R A S / R A F$ oncogenes as a risk factor for overall survival after CRS/ HIPEC in patients with colorectal PM. In addition, we present a novel score (BIOSCOPE) to stratify patients with colorectal PM before CRS/HIPEC.

The role of $R A S / R A F$ mutations in CRC is known as a surrogate marker for response rates to targeted chemotherapy ${ }^{30}$ and overall survival in the setting of palliative chemotherapy. ${ }^{31} \mathrm{In}$ patients with CRLM, RAS mutations are a prognostic factor ${ }^{32,33,34}$ and were included in a newly proposed modified version of the Clinical Risk Score for Prediction of Recurrence after resection of CRLM. $^{35}$ In contrast, its role as a predictive factor in patients 

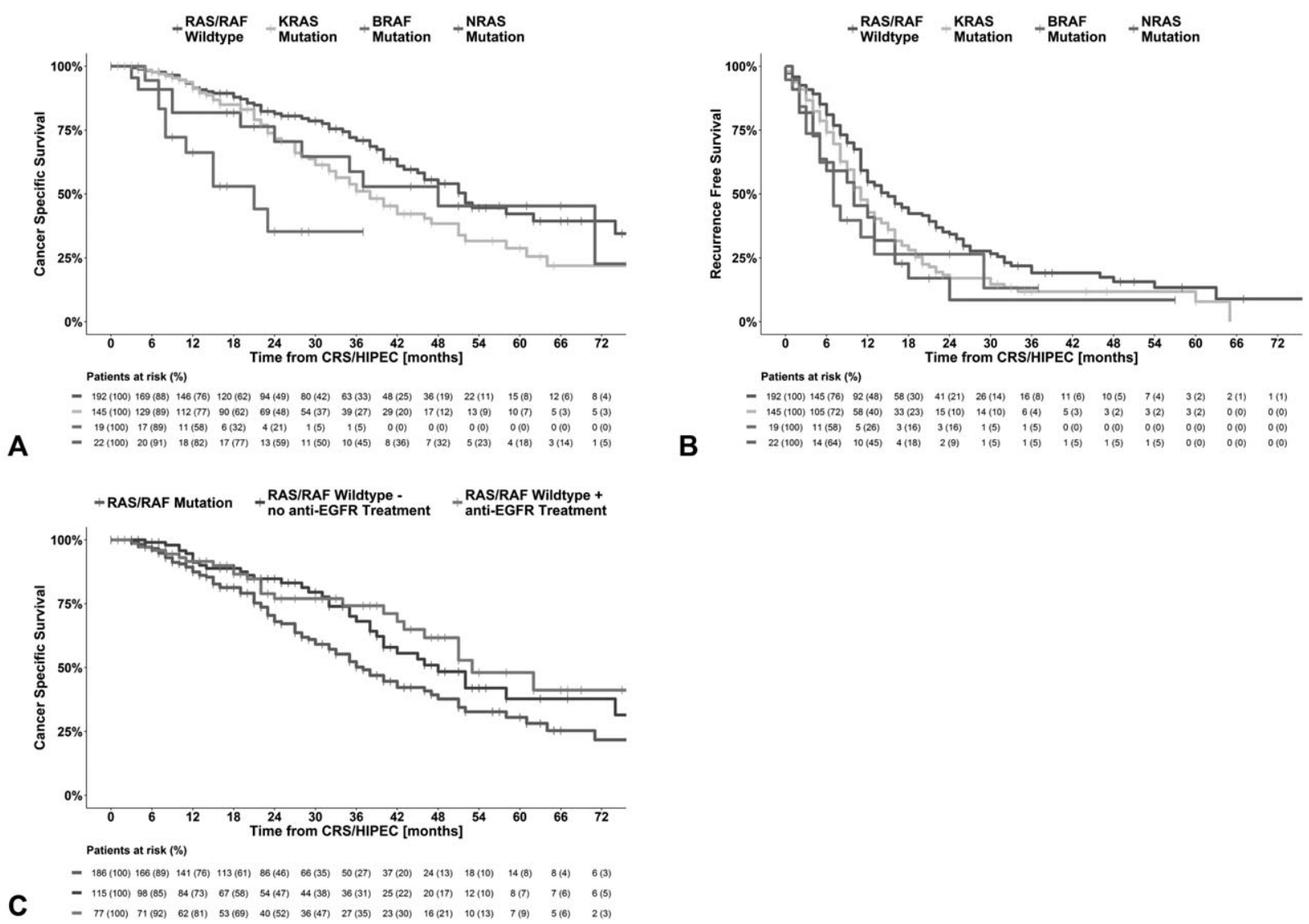

FIGURE 1. The influence of RAS/RAF mutations on outcomes after CRS/HIPEC. (A) While patients without RAS/RAF mutations show a median CSS of 52 months (Cl: 40-65), outcomes for patients with KRAS mutations (mCSS $38 \mathrm{mo}, \mathrm{Cl}: 31-46, P=0.036)$ and $B R A F$ mutations (mCSS of 21 mo, Cl: $9-26, P \leq 0.001$ ) were strongly impaired. No significant effect of NRAS mutations (mCSS: 51 mo, Cl: $19-80, P=0.533)$ on CSS was observed. (B) Similarly, median RFS was reduced by 4 months for patients with $K R A S$ mutations $(P=$ $0.007), 3$ months for NRAS mutations $(P=0.032)$ and 7 months $(P=0.051)$ for BRAF mutations compared with 15 months in unmutated patients. (C) The survival benefit of patients with wildtype RAS/RAF remained also if they were only treated with conventional chemotherapy without anti-EGFR treatment (mCSS $48 \mathrm{mo}, \mathrm{Cl}: 40-83$ ) compared with 37 months (Cl: $31-46)$ only in patients with mutant RAS/RAF $(P=0.028)$. Patients with wildtype RAS/RAF and treatment with anti-EGFR therapy showed a mCSS of 53 months $(\mathrm{Cl}: 42-64)$ without difference to wildtype patients with conventional chemotherapy only $(P=0.820)$.

undergoing surgery for PM, which are known to have a worse prognosis than liver or lung metastasis, is unclear. ${ }^{1}$ In the present study, we are able to demonstrate the specific influence of $R A S / R A F$ mutations on CSS and RFS in patients with PM from CRC undergoing CRS/HIPEC. We found impaired CSS and PFS after CRS/HIPEC for KRAS mutations, a heavy impact on CSS for BRAF mutations, whereas NRAS mutations did only impact on PFS in our cohort. Differences between $K R A S, N R A S$, and $B R A F$ mutations in postsurgical outcomes have so far not been described.

Our analysis confirmed other predictive factors, for example, the amount of peritoneal disease, assessed by the PCI, positive lymph node status of the primary tumor, and tumor grading. ${ }^{36,37}$ We consequently included PCI, N-, G-, and RAS/ $R A F$ mutational status in a Cox model, which showed good discrimination and calibration on the development as well as the validation cohort. We finally developed a score with a maximum of 12 points, which we termed BIOSCOPE (BIOlogical Score of COlorectal PEritoneal metastasis). When applied to both our cohorts, increasing score numbers strongly correlated with decreasing survival.
We then determined 4 groups based on points: BIOSCOPE A ( 0 risk points) represents patients with absent risk factors ( $\mathrm{PCI} \leq 10$, N0, G1-2, RAS/RAF wt). These patients can expect an excellent long-term outcome, reflected by mCSS of 70 and 65 months in our cohorts. BIOSCOPE B (1-3 risk points) reflects patients with moderate risk factors; these patients are able to reach mCSS of 50 and 39 months respectively, which is equal and above the mCSS of 40 to 45 months reported in recent analyses of patients undergoing CRS/HIPEC. ${ }^{6,7}$ BIOSCOPE C patients (4-7 points) profit from CRS/HIPEC with a mCSS of 33 and 25 months, which is clearly superior to the mCSS of 16.9 months in patients with PM treated systemically with modern targeted chemotherapy only. ${ }^{1}$ In contrast, BIOSCOPE D patients ( $\geq 8$ points) show a dismal survival of 13 (development) and 7 (validation) months only. CRS/HIPEC in these patients should be evaluated critically regarding possible complications and time for convalescence, and the decision for CRS/HIPEC should be made carefully on an individual basis.

We would like to acknowledge the limitations of our study. External validation is currently lacking and must be performed in an additional, independent patient cohort to confirm the value of the 

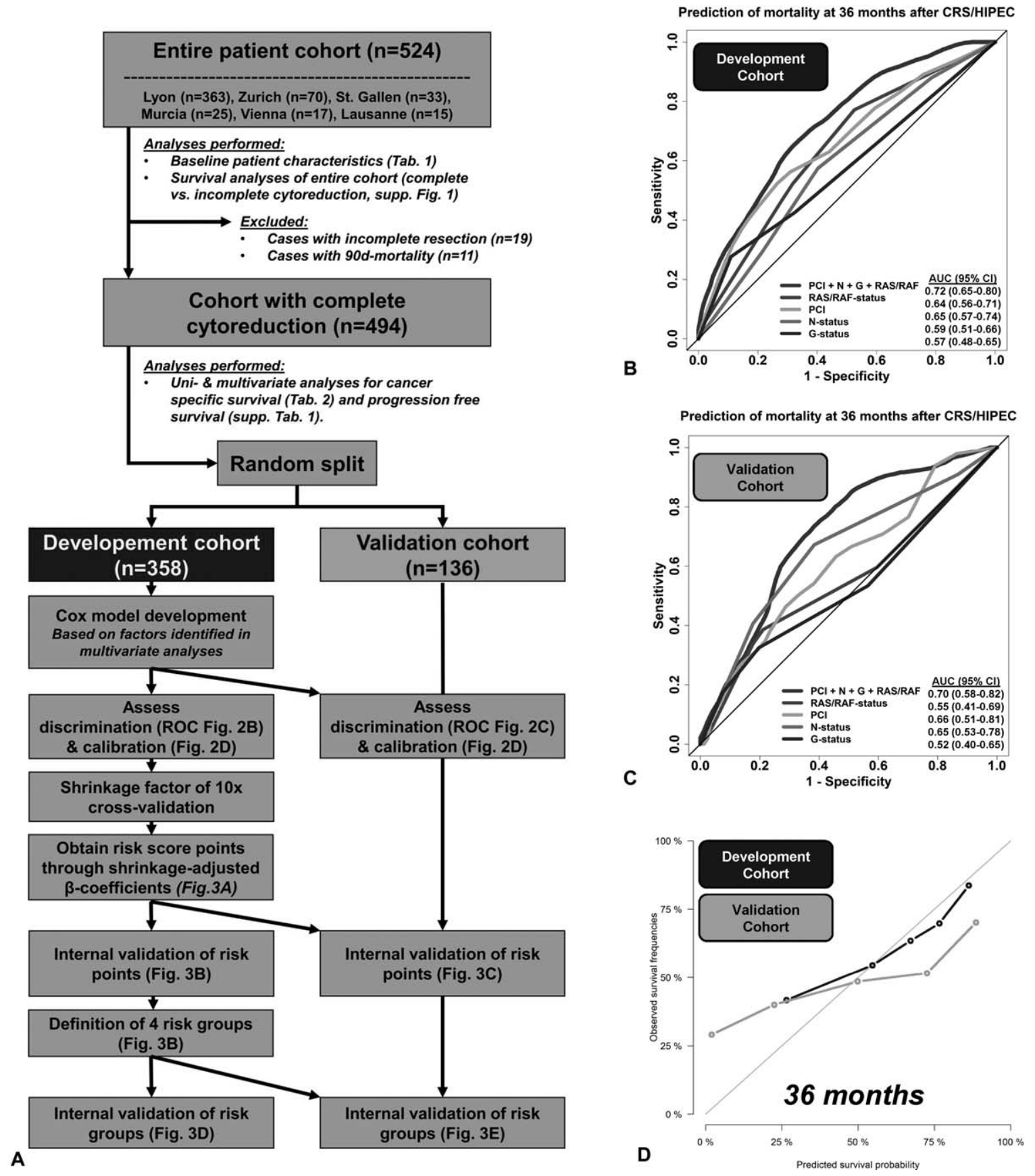

FIGURE 2. Discrimination and calibration of Cox regression model. (A) Methodological flow schema of patient cohort analyses and random splitting into development and validation cohort. (B) The Cox regression model with the 4 major predictive factors $\mathrm{PCl}$, $\mathrm{N}$-, G-, and RAS/RAF mutation status predicted survival at 3 years in the development cohort with a AUC value of 0.72 (Cl: $0.65-0.80$, $\left.R^{2}: 0.167\right)$ and showed superior discrimination compared to models consisting of the single predictive factors PCl (AUC: $0.65, R^{2}$ : 0.093 ), N-status (AUC: 0.59, $R^{2}: 0.03$ ), G-status (AUC: $0.57, R^{2}: 0.028$ ), or RAS/RAF mutational status (AUC: $0.64, R^{2}: 0.031$ ) only. (C) Consequent application of the model on the validation cohort confirmed the good discriminative ability with an AUC $=0.70$. (D) Observed survival frequencies and predicted survival probabilities at 3 years after CRS/HIPEC for quintiles of survival groups confirmed the strong calibration of the model in both cohorts. 


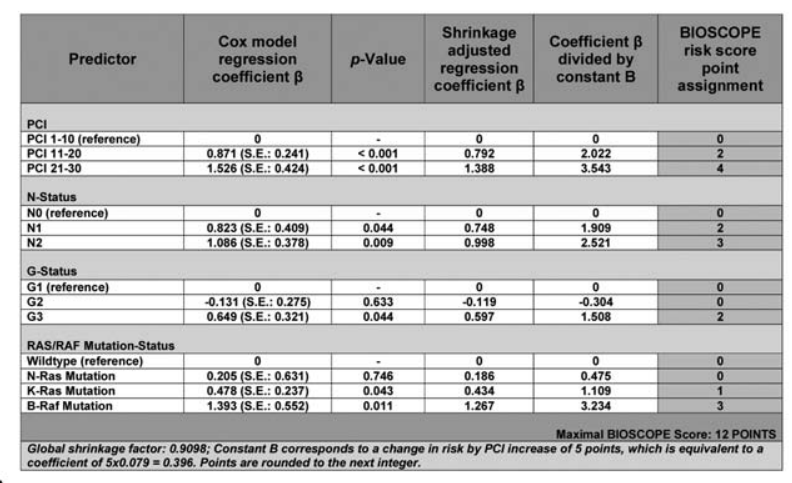

A

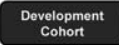

\begin{tabular}{|c|c|c|c|c|c|c|c|c|c|c|c|c|c|}
\hline $\begin{array}{l}\text { Bloscope } \\
\text { risk points }\end{array}$ & 0 & 1 & 2 & 3 & 4 & 5 & 6 & 7 & 8 & 9 & 10 & 11 & 12 \\
\hline $\begin{array}{c}\text { Nr. of } \\
\text { patients }\end{array}$ & 91 & 38 & 61 & 46 & 43 & 39 & 23 & 8 & 8 & 1 & 0 & 0 & 0 \\
\hline $\begin{array}{c}\text { Median } \\
\text { CSSS } \\
(195 \mathrm{C})\end{array}$ & $\begin{array}{c}70 \\
(38828)\end{array}$ & $\begin{array}{c}66 \\
135897)\end{array}$ & $\begin{array}{c}648965) \\
(4896)\end{array}$ & $\left|\begin{array}{c}49 \\
(28-7)\end{array}\right|$ & $\begin{array}{c}36 \\
(1268)\end{array}$ & $\begin{array}{lll}372.52) \\
(1)^{3}\end{array}$ & $\begin{array}{ll}33 & 2 \\
(16531) & 16 \\
\end{array}$ & $\begin{array}{c}23 \\
(19-40)\end{array}$ & $(14-28)$ & $\begin{array}{l}13 \\
(13-13)\end{array}$ & $\mathrm{NA}$ & $\mathrm{NA}$ & NA \\
\hline $\begin{array}{l}\text { Bloscope } \\
\text { risk group }\end{array}$ & A & & B & & & C & ; & & & & D & & \\
\hline
\end{tabular}

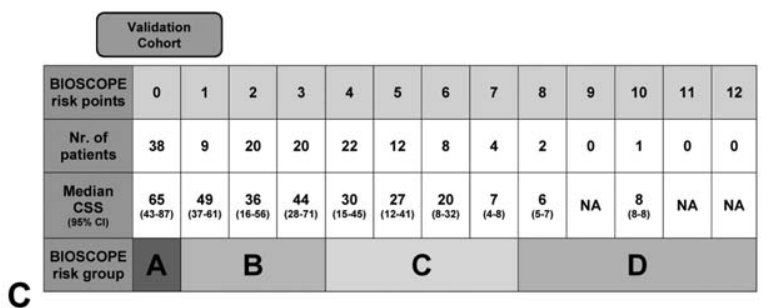

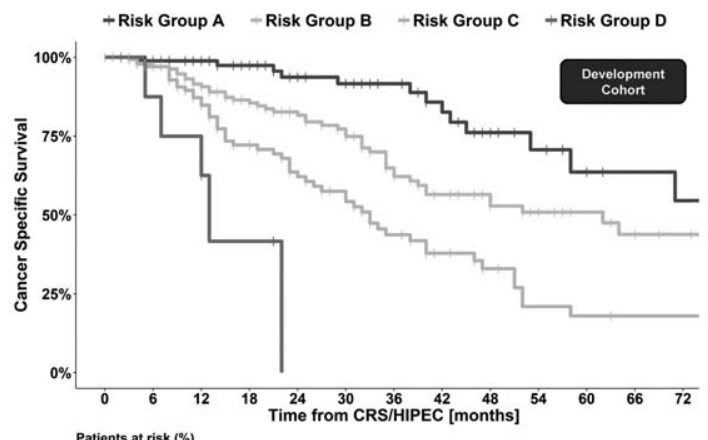

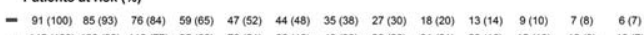

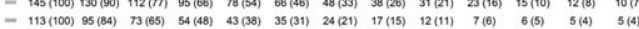
D
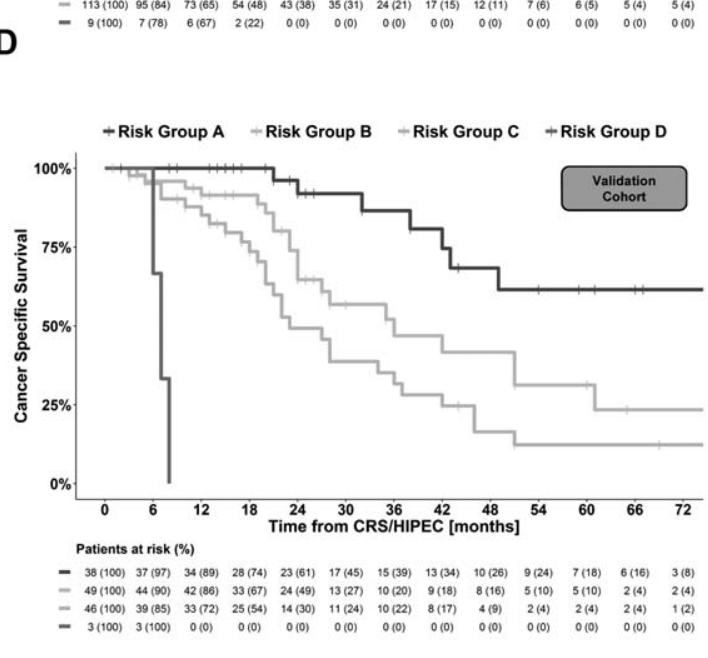

$\mathbf{E}$

FIGURE 3. BIOSCOPE risk score development \& internal validation. (A) A point-based risk score termed BIOSCOPE was developed by dividing shrinkage-adjusted $\beta$ regression coefficients through constant B. Points were rounded to the next integer. (B) Application of the score on our development cohort showed a consistent decrease of mCSS with increasing risk points. Four risk groups were defined based on similar survival. Patients with 0 risk points showed excellent long-term outcome with a mCSS of 70 months; patients with risk score between 1 and 3 had mCSS above the median of the whole cohort ( 43 mo) and were defined as risk group B. Patients with risk scores between 4 and 7 (risk group C) showed an acceptable survival, whereas patients with risk scores 8 and higher (risk group D) had a dismal long-term outcome similar or below the current survival reached by systemic palliative chemotherapy. (C) Risk point allocation to the validation cohort confirmed decreasing survival with increasing risk points. (D) Application of the risk score with consequent group allocation divided patients in the development cohort into groups with strongly divergent outcomes of 70 months CSS (Cl: 58-82) for group A, 55 months (Cl: 42-68) for group B, 33 months (Cl: 24-41) for group C, and 13 months (Cl: 5-24) for group $D(P \leq 0.001$ for all comparisons between the 4 groups). (E) The score confirmed its value for stratification of patient outcomes after $\mathrm{CRS} / \mathrm{HIPEC}$ by dividing patients in the validation cohort into 4 groups with mCSS of 65 months (CI: $43-87$ ) for group A, 39 months ( $\mathrm{Cl}$ : 27-51) for group $\mathrm{B}, 25(\mathrm{Cl}$ : 16-33) for group $\mathrm{C}$, and 7 (Cl: 5-9) for group $\mathrm{D}(P \leq 0.01$ for all comparisons between the 4 groups).

BIOSCOPE score for patient selection and classification. Internal validation in this study was performed by splitting the cohort into a development and validation cohort.

Preoperative determination of the PCI remains challenging, despite modern imaging by MRI, CT, or PET. Therefore, most centers performing CRS/HIPEC for CRC advocate to perform laparoscopy to assess the PCI before CRS/HIPEC. The PCI documented in our study was evaluated on explorative laparotomy. For the development of our score, we decided to separate the PCI in relatively large categories of 10 points to facilitate preoperative classification of patients based on laparoscopy.

Information regarding $R A S$ status of patients was based on the available retrospective data and no additional analysis of existing tumor tissue was performed. Most patients received testing of $K R A S$, but not NRAS or BRAF. Mutations of KRAS are the most frequent ${ }^{9,11}$ and normally mutually exclusive toward mutation of NRAS or $B R A F^{38-40}$ and therefore render further testing unnecessary. However, in cases of absent $K R A S$ mutation, it is nowadays recommended to consequently test for mutations in $N R A S,{ }^{9} B R A F,{ }^{9,10}$ and most recently PI3KCA (mutated in $3.5 \%$ of CRC), ${ }^{9,11}$ which all lead activation of EGFR downstream signals and render the patient resistant to anti-EGFR therapy. As additional retrospective testing of NRAS and BRAF was not possible, there might be the potential bias that patients without KRAS mutation were grouped in the wildtype group although being $N R A S$ or $B R A F$ mutated. Despite this, we consider this potential bias as negligible as rates of $R A S / R A F$ 
mutations in our patient sample compare well to the rates found in patients with CRC in systematic analyses. We therefore believe that our patient cohort represents an adequate sample of CRC patients.

\section{CONCLUSION}

Mutations of $K R A S$, and in particular $B R A F$, are negative prognostic factors in patients with PM of CRC undergoing CRS/ HIPEC. The novel BIOSCOPE score, including $R A S / R A F$ mutational status, PCI, and N- and G-status of the primary tumor, adequately predicts prognosis of patients, which can help to improve patient selection.

\section{ACKNOWLEDGMENTS}

The authors would like to express their deepest thanks and gratitude to Mrs. Isabelle Bonnefoy, Lyon, for the rapid and very careful data collection and extraction of the data of patients operated in Lyon.

\section{REFERENCES}

1. Franko J, Shi Q, Meyers JP, et al. Prognosis of patients with peritoneal metastatic colorectal cancer given systemic therapy: an analysis of individual patient data from prospective randomised trials from the Analysis and Research in Cancers of the Digestive System (ARCAD) database. Lancet Oncol. 2016;17:1709-1719.

2. Glehen O, Mohamed F, Gilly FN. Peritoneal carcinomatosis from digestive tract cancer: new management by cytoreductive surgery and intraperitoneal chemohyperthermia. Lancet Oncol. 2004;5:219-228.

3. Lehmann K, Eshmuminov D, Slankamenac K, et al. Where oncologic and surgical complication scoring systems collide: time for a new consensus for CRS/HIPEC. World J Surg. 2016;40:1075-1081.

4. Goéré D, Souadka A, Faron M, et al. Extent of colorectal peritoneal carcinomatosis: attempt to define a threshold above which HIPEC does not offer survival benefit: a comparative study. Ann Surg Oncol. 2015;22:2958-2964.

5. Elias D, Gilly F, Boutitie F, et al. Peritoneal colorectal carcinomatosis treated with surgery and perioperative intraperitoneal chemotherapy: retrospective analysis of 523 patients from a multicentric French study. J Clin Oncol. 2010;28:63-68.

6. Simkens GA, van Oudheusden TR, Luyer MD, et al. serious postoperative complications affect early recurrence after cytoreductive surgery and HIPEC for colorectal peritoneal carcinomatosis. Ann Surg Oncol. 2015;22:26562662.

7. Schneider MA, Eshmuminov D, Lehmann K. Major postoperative complications are a risk factor for impaired survival after CRS/HIPEC. Ann Surg Oncol. 2017;24:2224-2232.

8. Normanno N, Tejpar S, Morgillo F, et al. Implications for KRAS status and EGFR-targeted therapies in metastatic CRC. Nat Rev Clin Oncol. 2009; 6:519-527

9. Zhang J, Zheng J, Yang Y, et al. Molecular spectrum of KRAS, NRAS, BRAF and PIK3CA mutations in Chinese colorectal cancer patients: analysis of 1,110 cases. Sci Rep. 2015;5:18678.

10. Farina-Sarasqueta A, van Lijnschoten G, Moerland E, et al. The BRAF V600E mutation is an independent prognostic factor for survival in stage II and stage III colon cancer patients. Ann Oncol. 2010;21:2396-2402.

11. Løes IM, Immervoll H, Sorbye $\mathrm{H}$, et al. Impact of KRAS, BRAF, PIK3CA, TP53 status and intraindividual mutation heterogeneity on outcome after liver resection for colorectal cancer metastases. Int J Cancer. 2016;139:647-656.

12. Roth AD, Tejpar S, Delorenzi M, et al. Prognostic role of KRAS and BRAF in stage II and III resected colon cancer: results of the translational study on the PETACC-3, EORTC 40993, SAKK 60-00 trial. J Clin Oncol. 2010;28:466-474.

13. Tol J, Nagtegaal ID, Punt CJ. BRAF mutation in metastatic colorectal cancer. $N$ Engl J Med. 2009;361:98-99.

14. Kim IJ, Kang HC, Jang SG, et al. Oligonucleotide microarray analysis of distinct gene expression patterns in colorectal cancer tissues harboring BRAF and K-ras mutations. Carcinogenesis. 2006;27:392-404.

15. Ciardiello F, Tortora G. EGFR antagonists in cancer treatment. $N$ Engl J Med. 2008;358:1160-1174.

16. Cunningham D, Humblet Y, Siena S, et al. Cetuximab monotherapy and cetuximab plus irinotecan in irinotecan-refractory metastatic colorectal cancer. $N$ Engl J Med. 2004;351:337-345.
17. Jonker DJ, O'Callaghan CJ, Karapetis CS, et al. Cetuximab for the treatment of colorectal cancer. N Engl J Med. 2007;357:2040-2048.

18. Esquivel J, Lowy AM, Markman M, et al. The American Society of Peritoneal Surface Malignancies (ASPSM) Multiinstitution Evaluation of the Peritoneal Surface Disease Severity Score (PSDSS) in 1,013 patients with colorectal cancer with peritoneal carcinomatosis. Ann Surg Oncol. 2014;21:4195-4201.

19. Simkens GA, van Oudheusden TR, Nieboer D, et al. Development of a prognostic nomogram for patients with peritoneally metastasized colorectal cancer treated with cytoreductive surgery and HIPEC. Ann Surg Oncol. 2016;23:4214-4221.

20. Demey K, Wolthuis A, de Buck van Overstraeten A, et al. External validation of the prognostic nomogram (COMPASS) for patients with peritoneal carcinomatosis of colorectal cancer. Ann Surg Oncol. 2017;24:3604-3608.

21. Halkia E, Tsochrinis A, Vassiliadou DT, et al. Peritoneal carcinomatosis: intraoperative parameters in open (coliseum) versus closed abdomen HIPEC. Int J Surg Oncol. 2015;2015:610597.

22. Glehen O, Cotte E, Kusamura S, et al. Hyperthermic intraperitoneal chemotherapy: nomenclature and modalities of perfusion. J Surg Oncol. 2008; 98:242-246.

23. Sugarbaker PH. Intraperitoneal chemotherapy and cytoreductive surgery for the prevention and treatment of peritoneal carcinomatosis and sarcomatosis. Semin Surg Oncol. 1998;14:254-261.

24. Kang J. Missing Data in Longitudinal Covariates in Building a Cox Prediction Model: Overview and Some Practical Guidance. Biom Biostat Int J. 2015;2:00030.

25. Collins GS, Reitsma JB, Altman DG, et al. Transparent reporting of a multivariable prediction model for Individual Prognosis Or Diagnosis (TRIPOD): the TRIPOD statement. Br J Surg. 2015;102:148-158.

26. Demler OV, Paynter NP, Cook NR. Tests of calibration and goodness of fit in the survival setting. Stat Med. 2015;34:1659-1680.

27. Motwani SS, McMahon GM, Humphreys BD, et al. Development and validation of a risk prediction model for acute kidney injury after the first course of cisplatin. J Clin Oncol. 2018;36:682-688.

28. Sullivan LM, Massaro JM, D'Agostino RB Sr. Presentation of multivariate data for clinical use: the Framingham Study risk score functions. Stat Med. 2004;23:1631-1660.

29. Dindo D, Demartines N, Clavien P-A. Classification of surgical complications. Ann Surg. 2004;240:205-213.

30. Van Cutsem E, Köhne C-H, Hitre E, et al. Cetuximab and chemotherapy as initial treatment for metastatic colorectal cancer. $N$ Engl J Med. 2009; 360:1408-1417.

31. Andreyev HJ, Norman AR, Cunningham D, et al. Kirsten RAS mutations in patients with colorectal cancer: the 'RASCAL II' study. $\mathrm{Br} J$ Cancer. 2001;85:692-696.

32. Stremitzer S, Stift J, Gruenberger B, et al. KRAS status and outcome of liver resection after neoadjuvant chemotherapy including bevacizumab. Br J Surg. 2012;99:1575-1582.

33. Brudvik KW, Kopetz SE, Li L, et al. Meta-analysis of KRAS mutations and survival after resection of colorectal liver metastases. Br J Surg. 2015;102:1175-1183

34. Brudvik KW, Mise Y, Chung MH, et al. RAS mutation predicts positive resection margins and narrower resection margins in patients undergoing resection of colorectal liver metastases. Ann Surg Oncol. 2016; 23:2635-2643.

35. Brudvik KW, Jones RP, Giuliante F, et al. RAS mutation clinical risk score to predict survival after resection of colorectal liver metastases. Ann Surg. 2017. DOI: 10.1097/sla.0000000000002319.

36. Baumgartner JM, Tobin L, Heavey SF, et al. Predictors of progression in highgrade appendiceal or colorectal peritoneal carcinomatosis after cytoreductive surgery and hyperthermic intraperitoneal chemotherapy. Ann Surg Oncol. 2015;22:1716-1721.

37. Glehen O, Gilly FN, Boutitie F, et al. Toward curative treatment of peritoneal carcinomatosis from nonovarian origin by cytoreductive surgery combined with perioperative intraperitoneal chemotherapy: a multi-institutional study of 1,290 patients. Cancer. 2010;116:5608-5618.

38. Fleming M, Ravula S, Tatishchev SF, et al. Colorectal carcinoma: Pathologic aspects. J Gastrointest Oncol. 2012;3:153-173.

39. Koinuma K, Shitoh K, Miyakura Y, et al. Mutations of BRAF are associated with extensive hMLH1 promoter methylation in sporadic colorectal carcinomas. Int J Cancer. 2004;108:237-242.

40. Rajagopalan H, Bardelli A, Lengauer C, et al. Tumorigenesis: RAF/RAS oncogenes and mismatch-repair status. Nature. 2002;418:934. 


\section{DISCUSSANTS}

\section{Beat P. Müller (Heidelberg, Germany):}

I would like to thank and congratulate you for your innovative idea to develop the BIOSCOPE score, which should help us select patients for cytoreductive surgery and HIPEC for peritoneal metastasis originating from colorectal cancer in the future. Such a score is of great interest for both the surgical and oncological community. I very much appreciate the careful and thorough work you invested in this important idea.

First, I somewhat regret that you did not focus on this idea, and instead, spent most of the study introducing and discussing the importance of $R A S / R A F$ proto-oncogenes as a predictive factor, which results in the fact that the BIOSCOPE score is not even mentioned in the title of the manuscript. From a surgical point of view - and Annals of Surgery is a surgical journal - a score, which helps us to better select surgical patients, would be of major interest. Could you please comment on this?

Second, unfortunately, external validation is missing, and therefore, the score cannot be generally recommended as a valid prognostic stratification tool for the time being. This should be stated in the conclusions, abstract, mini-abstract, and manuscript. The opportunity to address this flaw would have been to use a nonrandom split sample (TRIPOD 2B) - for example, the Lion population - for validation. Why did you decide against this? Unfortunately, according to the TRIPOD statement, using a random split-sample does not improve score development and validation compared with TRIPOD $1 \mathrm{~B}$, which you applied in the first version of your manuscript.

Third, how can we know that the BIOSCOPE score provides any improvement, in terms of the prediction of prognosis and better patient selection, when compared with existing selection criteria, such as the peritoneal carcinosis index or others?

Fourth, how did you deal with other potential confounders and risk factors, which have not been mentioned so far, such as different histologies, chemotherapy regimes, surgical expertise, changed management during the investigated period and so on? This should at least be mentioned in the supplemental material.

\section{Response From Kuno Lehmann (Zurich, Switzerland):}

Thank you, Professor Müller, for your interesting questions. There is a big discussion around the biology among different types of metastases, for example, lung, liver, or peritoneal metastases. Our main goal here was to explore the biology of peritoneal metastasis. This is why I personally think that the identification of novel risk factors, for example, $K-R A S$ or $B-R A F$, is of major importance. BIOSCOPE is a useful tool that was developed afterward and will improve decision-making in the treatment of peritoneal metastasis.

Regarding your second question, the critical point here is external validation, and I acknowledge and agree with your point that this study needs independent external validations. I think that this is absolutely clear, and I'd be happy to welcome everyone, who would join us here and help us further develop BIOSCOPE.

Your third comment is very important. The peritoneal cancer index, PCI, is a known major predictor of survival in patients with peritoneal metastasis. The point here is that the surgeons operating these patients learned this already and tend to select patients in a quite narrow range of PCI. In our study, the median PCI ranged from 3 to 12 , and $70 \%$ of patients were below 10 . If the PCI range is narrowed in patients selected for surgery, then we need further parameters to predict outcomes, and I think our work can be of interest and help.

Regarding your last question, CRS/HIPEC was performed in all centers by qualified surgeons, and we did not observe any difference in survival among the institutions. We also analyzed factors, such as tumor histology or preoperative chemotherapy. For example, signet cell histology was significant in the univariate analysis, but not in the multivariate analysis. We did not find any significance for preoperative chemotherapy, which was applied in $80 \%$ of our patients.

\section{Christiane Bruns (Cologne, Germany):}

Basically, with the BIOSCOPE score, you tried to gain further information to select patients for cytoreductive surgery and HIPEC, with peritoneal metastasis originating from colorectal cancer beyond the PCI score. Did you integrate the location of the primary tumor as a confounder within the BIOSCOPE score, since the distribution of the mutations of the $R A S / R A F$ proto-oncogenes is different? This automatically leads me to the next question: Did you integrate the impact of different chemotherapies and biologicals in the development of the BIOSCOPE score?

\section{Response From Kuno Lehmann (Zurich, Switzerland):}

Thank you, Professor Bruns, for your interesting question. We looked at the influence of the site of the primary tumor, but did not identify a predictive role. We also looked at different types of chemotherapies and the addition of VEGF antibodies, which did not have a significant impact. Importantly, the addition of EGFR antibodies did not improve the outcome, even in $K-R A S$ wild-type patients.

\section{Olivier Turrini (Marseille, France):}

Congratulations on this very interesting work. I have one question. Did you look at the original location of the mutation? Was it on Codon 12 or 13 because it has been shown in liver metastases that recurrence rate was higher, when a $K$-RAS mutation occurred on Codon 13 ?

\section{Response From Kuno Lehmann (Zurich, Switzerland):}

Thank you, Professor Turrini, that is a very important question. Unfortunately, we do not have this data. However, this would be an interesting study for the future. 